\title{
MEDICIÓN DE LA ADHERENCIA AL LAVADO DE MANOS, SEGÚN LOS CINCO MOMENTOS DE LA OMS
}

\section{MEASUREMENT OF HANDWASHING ADHERENCE, ACCORDING TO FIVE MOMENTS OF THE WHO}

\section{TITULO CORTO: MEDICIÓN DE LA ADHERENCIA AL LAVADO DE MANOS}

\section{Oscar Alberto Villegas-Arenas ${ }^{1}$, Jaime Gómez ${ }^{2}$, Jorge Uriel-López ${ }^{3}$, Richard Nelson Román ${ }^{4}$, Jazmín Eliana Villa ${ }^{5}$, Jéssica Botero ${ }^{6}$, Nathalia García ${ }^{6}$}

Recibido en mayo 05 de 2016

Aceptado en noviembre 04 de 2016

Publicado en línea en mayo 15 de 2017

\section{Resumen}

El contacto con objetos y personas en el entorno hospitalario favorece la propagación de gérmenes que amenazan a los pacientes; el lavado de manos es una estrategia clave para prevenir infecciones. En este estudio, transversal y cuantitativo, se midió la adherencia al lavado de manos en personal ambulatorio y de urgencias de Assbasalud ESE, según los cinco Momentos de la Higiene de las Manos de la OMS, y se evaluó el impacto después de impartir capacitación. Lo anterior se realizó en tres fases: precapacitación, capacitación y poscapacitación, las cuales contaron con el aval del Comité de Calidad de la IPS. En la formación se tuvieron en cuenta las etapas de la higiene de manos, el uso de esmaltes y joyas y la pertinencia de las sustancias empleadas para la asepsia. La adherencia obtenida al lavado de manos en la primera fase fue del $61,8 \%$ y en la segunda, del 78,3\%. Estos resultados son positivos con relación a estudios internacionales. Se concluye que el lavado de manos, valorado en 78,3\% durante el cuarto trimestre de 2013, es una excelente estrategia para prevenir infecciones hospitalarias.

Palabras clave: Desinfección de las manos; infección hospitalaria; antiinfecciosos locales; seguridad del paciente.

\section{Abstract}

Contact with objects and people in the hospital environment promotes the spread of germs that threaten patients; hand washing is a key strategy to prevent infections. Adherence to hand washing of health care officials was measured according to the Five Moments of Hand Hygiene of the WHO (World Health Organization). This transversal and quantitative study evaluated the adherence to hand washing in outpatient and emergency personnel of Assbasalud ESE, as well as the impact after receiving training. It was conducted in three phases: pre-training, training and post-

1. Magister en Educación Docencia. Profesor titular tiempo parcial. Universidad de Manizales. Colombia. Correo: oscarvillegasa@hotmail.com

2. Especialista en Gerencia de Servicios Sociales. Médico tiempo completo. Assbasalud ESE. Manizales, Colombia. Correo: jaime.gomez@assbasalud.gov.co

3. Especialista en Gerencia de Servicios Sociales. Médico tiempo completo. Assbasalud ESE. Manizales, Colombia. Correo: jorge.lopez@assbasalud.gov.co

4. Magister en Salud Pública. Ingeniero tiempo completo. Assbasalud ESE. Manizales, Colombia. Correo: richard.roman@assbasalud.gov.co

5. Especialista en Gerencia de Calidad. Enfermera de Calidad. Assbasalud ESE. Manizales, Colombia. Correo: jazmin.villa@assbasalud.gov.co

6. Estudiantes de Enfermería VIII semestre. Universidad Católica de Manizales, Colombia. Correos: jessikbotero@hotmail.com; natysgarcia_77@hotmail.com 
training. The final sample was 128 employees in nine bank suite's (30\%). The adhesion measurement was approved by the Executive Quality Committee of Assbasalud ESE. The factors that were evaluated were: hand hygiene, use of nail polish and use of jewels, as well as the relevance of the substances used for aseptic. The overall adherence of hand washing in phase one was of $61.8 \%$ and for the second phase, of $78.3 \%$. These are positive results with reference to national and international studies. It was concluded that hand washing, valued at $67.1 \%$ during the third quarter of 2013, is an excellent strategy to reinforce patient safety and to prevent hospital infections.

Keywords: Hands disinfection; hospital infection; local anti-infectives; patient safety.

\section{INTRODUCCIÓN}

$\mathrm{L}$ atención limpia es una acción de gran impacto para evitar infecciones asociadas, para prestar servicios de salud con calidad, para dar seguridad a los pacientes y "para salvar vidas". La adherencia al lavado de manos en procedimientos limpios es fundamental para cumplir estos objetivos. Esta constituye una buena estrategia costoefectiva para prevenir enfermedades; "manos limpias salvan vidas" es el lema de la Organización Mundial de la Salud (OMS) y "hospitales limpios salvan más vidas" sentencia Guillermo L. Lossa, director del programa VIHDA (Programa Nacional de Epidemiología y Control de Infecciones Hospitalarias, Argentina) ${ }^{1-4}$. Sin embargo, esta acción suele tener baja adhesión en los hospitales por falta de compromiso, de capacitación, de insumos o por "escaso tiempo" 1.

Las infecciones impactan la morbilidad y la mortalidad, sobre todo en ancianos y pacientes debilitados. Las infecciones nosocomiales afectan al $5 \%$ de los pacientes ingresados a los hospitales 5 . Aunque los gérmenes se pueden propagar por casualidad, es más probable que se transmitan cuando se toca al paciente después de haber palpado superficies u objetos contaminados ${ }^{6}$. Por ello, el reto mundial liderado por la OMS, "atención limpia es atención más segura”, está centrado en mejorar las prácticas de higiene de las manos durante la atención sanitaria ${ }^{2,3}$.

Las infecciones derivadas de la prestación de servicios de salud comprometen a muchas personas, aumentan los costos de la atención, causan nuevas lesiones, convalecencias prolongadas e incluso la muerte. La higiene de las manos es una acción sencilla que aporta de manera positiva a la reducción de riesgos prevenibles, convirtiéndose en indicador de calidad de la atención ${ }^{2,3}$.

En Estados Unidos, las consecuencias onerosas de las infecciones hospitalarias han aumentado con costos entre 4500 y 5700 millones de dólares al año; igual ocurre en Inglaterra donde estas cuestan mil millones de euros al año $0^{4}$. Al menos $7 \%$ de los pacientes hospitalizados en los países desarrollados y $10 \%$ en países pobres contraen infecciones relacionadas con la atención sanitaria ${ }^{7,8}$.

El problema es global y lo soportan estudios como el publicado en la Revista Latinoamericana de Enfermería en 2008, donde se evidencia que no hay conciencia en la profilaxis de lavar las manos o en asepsia con alcohol glicerinado ${ }^{7,8}$. En España se registran más de 300.000 casos al año de contagios en hospitales ${ }^{9}$; en Argentina las infecciones intrahospitalarias provocan 17.000 muertes y casi 100.000 episodios infecciosos al año, más que los accidentes de tránsito (3699 víctimas/año) y que las ocasionadas por armas de fuego (1922 víctimas/año) ${ }^{10,11}$. La mitad o más de las infecciones relacionadas con la atención sanitaria son prevenibles si los cuidadores se limpian debidamente las manos durante la atención asistencial. El paciente debe exigir al personal sanitario que lo va tocar que se limpie las manos y agradecerle cuando lo haga ${ }^{1,11}$.

El propósito de este estudio es evaluar el impacto del lavado de las manos en funcionarios asistenciales, como componente del Programa de Seguridad del Paciente implementado en Assbasalud ESE, de acuerdo a los cinco momentos de la Higiene de las Manos de la OMS. 


\section{MATERIALES Y MÉTODOS}

Estudio de corte cuantitativo y transversal aplicado a personal asistencial en varias sedes de Assbasalud ESE (IPS pública que presta atención de baja complejidad a la población subsidiada de Manizales-Colombia). Se realizó la prueba piloto en mayo de 2013 y el trabajo de campo, en el tercer trimestre del mismo año. El proceso fue realizado por estudiantes del último semestre de Enfermería de la Universidad Católica de Manizales, a través de visitas no anunciadas y en horario laboral diurno (mañanas y tardes), evitando los fines de semana y festivos. Se hizo la evaluación durante la rutina diaria del personal: se observó discretamente el lavado de manos en los diferentes momentos y se registró la información para posterior tabulación y análisis de los datos. Se realizó retroalimentación "en caliente" en cada visita y una socialización en los encuentros administrativos; esto sirvió de garante de la fidelidad del proceso.

La muestra incluyó 128 funcionarios asistenciales (54\% del total), en nueve sedes; $100 \%$ de las clínicas (tres); y $20 \%$ de los centros de salud, tres del área urbana y tres del área rural. Se trata de una muestra por conveniencia que permitió estimar el comportamiento global de la IPS.

Las variables objeto de evaluación fueron: lavado de manos con agua y jabón; lavado con alcohol glicerinado; uso de joyas y esmaltes; adherencia al lavado de manos según los cinco Momentos de la Higiene de las Manos de la $\mathrm{OMS}^{2}$, a saber: "1. Antes del contacto con el paciente, 2. Antes de realizar una tarea aséptica, 3. Después del riesgo de exposiciones a fluidos corporales, 4 . Después del contacto con el paciente, y 5 . Después del contacto con el entorno del paciente". Este último punto fue evaluado solo en el área hospitalaria.

Respecto a los criterios de inclusión, se tuvieron en cuenta los Funcionarios del área hospitalaria y ambulatoria. Criterios de exclusión: Proceso incompleto. Las observaciones se llevaron a cabo en días sucesivos y por cada fase objeto de verificación, precapacitación y poscapacitación. El instrumento de captura de la información es institucional y es aprobado por el Comité de Calidad de Assbasalud E.S.E., se basa en las directrices emanadas de la Dirección Territorial de Salud de Caldas y se ciñe a los cinco momentos de la Higiene de las Manos de la OMS. Se tuvo en cuenta, además, la norma interna de julio de 2013 sobre el no uso de esmaltes, anillos, relojes y joyas, y sobre los insumos recomendados para la higiene, como agua y jabón o alcohol glicerinado. Los análisis estadísticos se realizaron a través del programa Microsoft Excel 2007. La capacitación se desarrolló durante las rondas administrativas-asistenciales que se llevan a cabo semanalmente en todas las sedes, a través de campañas informativas internas, pendones, posters e información suficiente en la plataforma virtual (INTRANET). Además de esto, en cada punto de aseo de manos se ubicó un plegable explicando el correcto procedimiento.

\section{Declaración sobre aspectos éticos}

Según la resolución 8430 de $1993^{12}$, esta es una investigación sin riesgo biológico, psicosocial y cultural, que además respeta los Principios Generales de la Declaración de Helsinski ${ }^{13}$, en especial aquellos que invitan a "velar solícitamente por la salud de mi paciente" y a "considerar lo mejor para el paciente cuando preste atención médica".

\section{RESULTADOS}

Distribución de los funcionarios participantes en el seguimiento del lavado de manos: el área urbana representó un $83,5 \%$ y el área rural, un $16,5 \%$. Los participación en las clínicas fue de $66,4 \%$ y en los centros de salud, de 33,6\% (Tabla 1).

En la fase de precapacitación varias sedes $(2,5,7,8$ y 9) lograron adherencia global igual o superior al $75 \%$ y otras sedes (3 y 4) presentaron omisión del lavado de manos superior al 50\%. En la fase poscapacitación, algunas sedes $(2,5,6,7$ y 8$)$ mostraron una adherencia superior al $90 \%$, y otras ( 3 y 9), mayor omisión al lavado de manos -entre el $25 \%$ y $50 \%$-. Al relacionar las fases de pre y poscapacitación, se obtuvo un consolidado que revela un $16,5 \%$ de mejoría, así como más adherencia a la asepsia con agua y jabón que a la realizada con alcohol glicerinado (Tablas 2 y 3 ). 
Tabla 1. Adherencia al lavado de manos en funcionarios de Assbasalud ESE, distribuidos según área de trabajo y puntos de atención. Manizales 2013.

\begin{tabular}{|l|c|c|c|c|}
\hline \multicolumn{1}{|c|}{ Tipo y puntos de atención } & Sede & Área & $\begin{array}{c}\text { Funcionarios } \\
\text { evaluados }\end{array}$ & Porcentaje \\
\hline Clínica Centro Piloto & 1 & Urbana & 20 & 15,6 \\
\hline Centro de salud Cervantes & 2 & Urbana & 6 & 4,7 \\
\hline Clínica San Cayetano & 3 & Urbana & 20 & 15,6 \\
\hline Clínica La Enea & 4 & Urbana & 7 & 5,5 \\
\hline Centro de salud Bajo Tablazo & 5 & Rural & 6 & 4,7 \\
\hline Centro de salud La Linda & 6 & Rural & 10 & 7,8 \\
\hline Centro de salud El Carmen & 7 & Urbana & 6 & 4,7 \\
\hline Centro de salud El Bosque & 8 & Urbana & 128 & 6,3 \\
\hline Centro de salud Cuchilla del Salado & 9 & Rural & 600 \\
\hline
\end{tabular}

Fuente: Elaboración propia

Tabla 2. Valoración de la técnica de lavado de manos, según los insumos utilizados, antes de la capacitación. Assbasalud ESE Manizales 2013.

\begin{tabular}{|c|c|c|c|c|}
\hline \multicolumn{5}{|c|}{ PRECAPACITACIÓN } \\
\hline Sede & $\begin{array}{c}\text { Porcentaje de } \\
\text { adherencia al lavado } \\
\text { (alcohol glicerinado) }\end{array}$ & $\begin{array}{c}\text { Porcentaje de } \\
\text { adherencia al lavado } \\
\text { (agua y jabón) }\end{array}$ & $\begin{array}{c}\text { Porcentaje de } \\
\text { adherencia global }\end{array}$ & $\begin{array}{c}\text { Porcentaje } \\
\text { consolidado } \\
\text { omisiones }\end{array}$ \\
\hline 1 & 18,1 & 54,6 & 72,7 & 27,3 \\
\hline 2 & 0 & 75 & 75 & 25 \\
\hline 3 & 7,42 & 42,3 & 49,8 & 50,2 \\
\hline 4 & 8,8 & 26,2 & 35 & 65 \\
\hline 5 & 12,5 & 62,5 & 75 & 25 \\
\hline
\end{tabular}


Continuación Tabla 2.

\begin{tabular}{|c|c|c|c|c|}
\hline \multicolumn{5}{|c|}{ PRECAPACITACIÓN } \\
\hline Sede & $\begin{array}{c}\text { Porcentaje de } \\
\text { adherencia al lavado } \\
\text { (alcohol glicerinado) }\end{array}$ & $\begin{array}{c}\text { Porcentaje de } \\
\text { adherencia al lavado } \\
\text { (agua y jabón) }\end{array}$ & $\begin{array}{c}\text { Porcentaje de } \\
\text { adherencia global }\end{array}$ & $\begin{array}{c}\text { Porcentaje } \\
\text { consolidado } \\
\text { omisiones }\end{array}$ \\
\hline 6 & 0 & 66,7 & 66,7 & 33,3 \\
\hline 7 & 20 & 55 & 75 & 25 \\
\hline 8 & 16,7 & 83,3 & 100 & 0 \\
\hline 9 & 9,2 & 68,3 & 77,5 & 22,5 \\
\hline PROMEDIO & $\mathbf{1 2 , 2}$ & 49,6 & 61,8 & 38,2 \\
\hline
\end{tabular}

Fuente: Elaboración propia

Tabla 3. Valoración de la técnica de lavado de manos, según los insumos utilizados, después de la capacitación. Assbasalud ESE Manizales 2013.

\begin{tabular}{|c|c|c|c|c|c|}
\hline \multicolumn{6}{|c|}{ POSCAPACITACIÓN } \\
\hline Sede & $\begin{array}{c}\text { Porcentaje de } \\
\text { adherencia al } \\
\text { lavado (alcohol } \\
\text { glicerinado) }\end{array}$ & $\begin{array}{c}\text { Porcentaje de } \\
\text { adherencia al } \\
\text { lavado (agua y } \\
\text { jabón) }\end{array}$ & $\begin{array}{l}\text { Porcentaje de } \\
\text { adherencia } \\
\text { global }\end{array}$ & $\begin{array}{l}\text { Porcentaje } \\
\text { consolidado } \\
\text { omisiones }\end{array}$ & $\begin{array}{c}\text { Cambio de } \\
\text { porcentaje de } 1^{a} \\
\text { a } 3^{a} \text { fase }\end{array}$ \\
\hline 1 & 35 & 50 & 85 & 15 & 12,3 \\
\hline 2 & 8,33 & 91,7 & 100 & 0 & 25 \\
\hline 3 & 18,5 & 28,4 & 46,9 & 53,1 & $-2,9$ \\
\hline 4 & 22,5 & 57,5 & 80 & 20 & 45 \\
\hline 5 & 15 & 75 & 90 & 10 & 15 \\
\hline 6 & 33,3 & 58,3 & 91,7 & 8,3 & 25 \\
\hline 7 & 10 & 85 & 95 & 5 & 2 \\
\hline 8 & 25 & 75 & 100 & 0 & 0 \\
\hline 9 & 25 & 50 & 75 & 25 & $-2,5$ \\
\hline PROMEDIO & 23,8 & 54,4 & 78,3 & 21,7 & 16,5 \\
\hline
\end{tabular}

Fuente: elaboración propia 
En la etapa de precapacitación, las sedes 7 y 8 incumplieron la directriz de no uso de esmaltes y joyas, con un resultado igual o superior al $30 \%$. Otras sedes $(1,2,4,5$ y 9) presentaron un mayor cumplimiento, con resultados superiores al 91,7\%. En la fase de poscapacitación, las sedes 6 y 9 mostraron menor cumplimiento a la directriz de no uso de esmaltes y joyas con un resultado igual o superior al $25 \%$. Las sedes 2, 3, 4 y 8 cumplieron esta directriz con un resultado superior al $87,5 \%$ (Tabla 4). La adherencia global promedio al lavado de manos alcanzó un 78,3\%.

Tabla 4. No uso de esmaltes y joyas, pre y poscapacitación. Assbasalud ESE Manizales 2013.

\begin{tabular}{|c|c|c|c|c|}
\hline \multirow{3}{*}{ SEDE } & Precapacitación & Precapacitación & Posc apacitación & \multirow{2}{*}{$\begin{array}{c}\text { Cambio de } \% \text { de } 1^{a} \\
\text { a } 3^{\text {a }} \text { fase }\end{array}$} \\
\hline & \% consolidado & \% consolidado & \% consolidado & \\
\hline & incumple & cumple & incumple & \\
\hline 1 & 7,1 & 92,9 & 18,8 & -11.7 \\
\hline 2 & 0 & 100 & 0 & Estable \\
\hline 3 & 16,7 & 83,3 & 4,2 & 12.5 \\
\hline 4 & 0 & 100 & 12,5 & -12.5 \\
\hline 5 & 0 & 100 & 20 & -20 \\
\hline 6 & 16,7 & 83,3 & 33,3 & -16.6 \\
\hline 7 & 30 & 70 & 20 & 10 \\
\hline 8 & 33,3 & 66,7 & 0 & 33.3 \\
\hline 9 & 8,3 & 91,7 & 25 & -16.7 \\
\hline PROMEDIO & 12,3 & 87,7 & 13,3 & -1 \\
\hline
\end{tabular}

Fuente: Elaboración propia

En general, al relacionar las fases pre y poscapacitación, hubo una disminución global del $1 \%$, lo que quizás obedezca a lo reciente de la norma institucional y a condiciones particulares de tipo médico en algunos funcionarios.

Al evaluar el primer momento de la OMS - "lavado de manos antes del contacto con el paciente”-, se encontró una adherencia, en promedio, del $68,35 \%$ en la fase previa y de $81,63 \%$ en la tercera fase, con un incremento del $13,28 \%$. Con respecto al cuarto momento de la OMS - "después del contacto con el paciente" -, se observó una adherencia del $65,38 \%$ en la $1^{\circ}$ fase y del $65,22 \%$ en la $3^{\circ}$ fase.
El estudio del segundo momento de la OMS -"antes de hacer una tarea aséptica"-, mostró que, en promedio, un $55,8 \%$ en la primera fase y un $85,4 \%$ en la tercera fase cumplieron exitosamente la actividad. En cuanto al tercer momento -"después de tener contacto con secreciones o fluidos corporales"-, los resultados muestran un cumplimiento del $62,1 \%$ en la primera fase y de $82,9 \%$ (en promedio).

Respecto al quinto momento - "después del contacto con el entorno del paciente"-, la adherencia fue, en promedio, del $87,95 \%$ y del $89,3 \%$ para antes y después de la capacitación. Ambas cifras son muy satisfactorias (Tabla 5). 
Tabla 5. Valoración de los cinco Momentos de la Higiene de las Manos, en la pre y poscapacitación, diferenciado según sedes seleccionadas. Assbasalud ESE Manizales 2013.

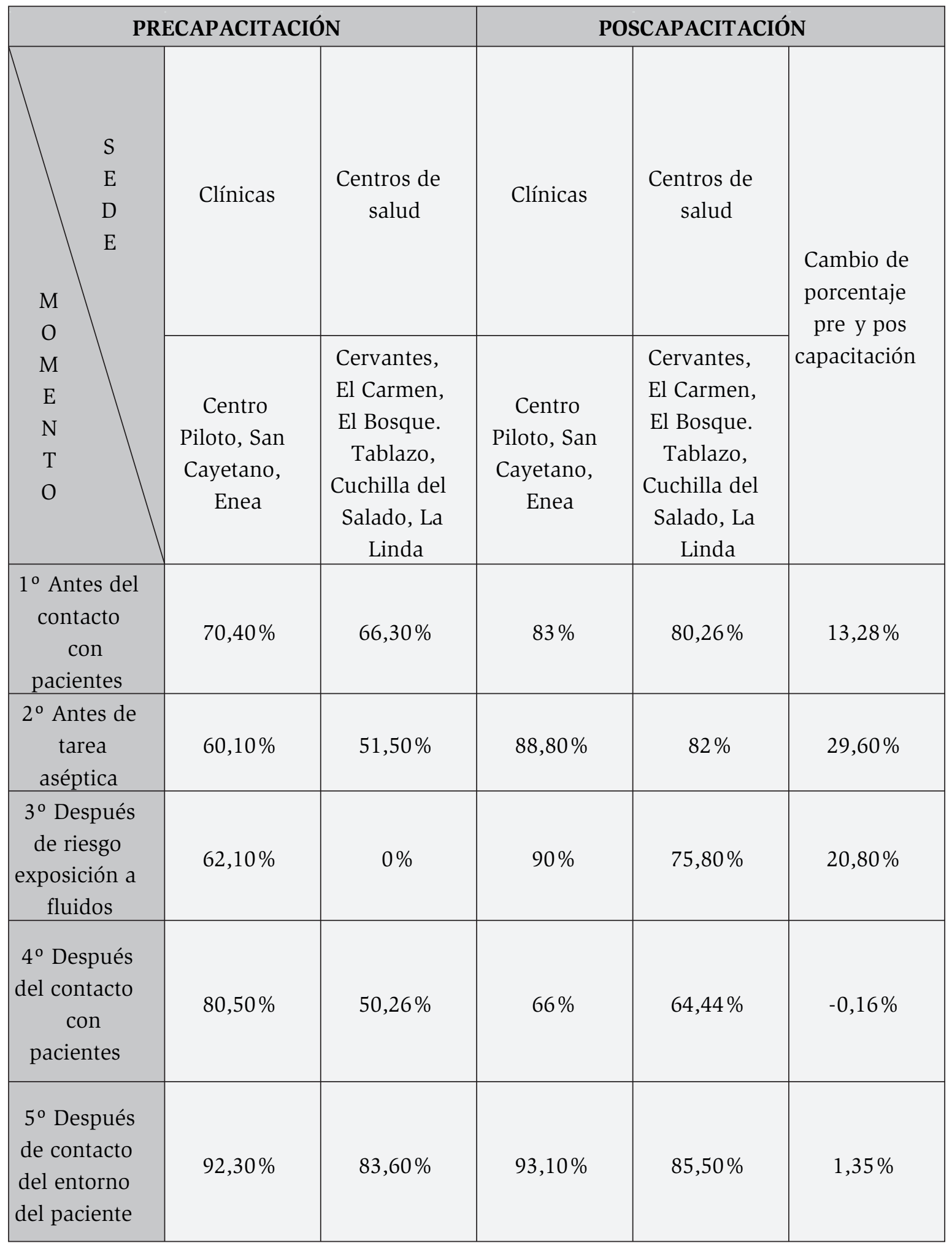

Fuente: Elaboración propia. 


\section{DISCUSIÓN}

El lavado de manos constituye para Assbasalud ESE una importante estrategia para la prevención de infecciones y una acción trascendental para fortalecer la cultura de seguridad del paciente, cuantificada en el tercer trimestre de 2013 en 78,3\%.

Para el presente estudio, los promedios hallados respecto a la adherencia al lavado de las manos en los funcionarios asistenciales, evaluados de acuerdo al segundo momento de la OMS, que cumplían exitosamente con esta actividad, fueron del $55,8 \%$ en la primera fase y del $85,4 \%$ en la tercera. Para el tercer momento de la OMS, los resultados promedio fueron del $62,1 \%$ en la primera fase y de $82,9 \%$ en la tercera fase. Respecto a estos dos Momentos de la OMS, se obtuvieron mejores porcentajes que los que se evidencian en la publicación de Ardila y Muñoz ${ }^{8}$ en 2009, donde describen que $57,6 \%$ cumplieron los parámetros impuestos y un $42,4 \%$, no lo hicieron.

Entre la primera y la tercera fase, se evidenció un incremento de casi el doble en el porcentaje obtenido en la adherencia al lavado de manos (16,5\%), respecto a un estudio multimodal de higiene de manos llevado a cabo en un hospital pediátrico de tercer nivel en México en donde el incremento fue del 8,89\% entre la primera y la segunda observación ${ }^{14}$.

En relación con el primer momento de la OMS, se encontró una adherencia promedio del $68,35 \%$ en la fase previa y de $81,63 \%$ en la posterior, con incremento del $13,28 \%$. Con respecto al cuarto momento de la OMS, referido como "después del contacto con el paciente", se observó una adherencia del $65,38 \%$ en la primera fase y del $65,22 \%$ en la tercera, sin variaciones entre ambos.

Al comparar estos datos con los del estudio de Sánchez et $\mathrm{al}^{15}$ en 2007, puede decirse que hubo mejor adherencia en el primer momento de la OMS. En dicho estudio se informa de una adherencia del 12,4\% antes del contacto con el paciente y del $48,8 \%$ en la segunda observación; así mismo, allí se habla de una adherencia del 42,1\% en la primera observación después del contacto con el paciente y del $68,9 \%$ en la segunda observación de ese momento. En el presente estudio, en la evaluación después del contacto con el paciente, la cifra de la primera observación es mayor a la del estudio de Sánchez et al ${ }^{15}$ y, levemente inferior al dato de la segunda observación. Los resultados obtenidos de la evaluación de la adherencia de lavado de manos, en el presente estudio -con un dato del $61,8 \%$ en la fase precapacitación y del $78,3 \%$ en la fase poscapacitación-, se pueden comparar con los publicados por Sánchez et al ${ }^{15}$ en 2007, donde informa una adherencia global a la higiene de manos del $40,5 \%$ en la primera observación y del 46,2\% en la segunda, calificada como una diferencia estadísticamente significativa ${ }^{15}$. La adherencia global al lavado de manos, reportada en el actual estudio, es menor que la del estudio referenciado.

Al revisar los archivos del Instituto de Salud Pública de México ${ }^{16}$, en 2010 se documenta que en las unidades de cuidados intensivos pediátricas se cumple en $34 \%$ la directriz del lavado de manos. En la publicación de Pessoa et $\mathrm{al}^{17}$ se reporta un $42 \%$ de adherencia a esta práctica entre trabajadores de la salud, valor cercano al encontrado como adherencia global del presente trabajo (49,6\%). Así mismo, en la publicación del Hospital Universitario Queen Mary en Hong-Kong, la adherencia al lavado de manos en el personal de salud fue de un $40 \%$, porcentaje de los más altos según la literatura consultada.

Es posible ver, al correlacionar los datos anteriores con los obtenidos en el presente estudio, que estos últimos van a la par con las estadísticas internacionales, teniendo en cuenta que la evaluación se desarrolló en una entidad de primer nivel de complejidad ${ }^{18}$. Con alta probabilidad, se puede afirmar que este tipo de medidas preventivas evitarán casos severos de sepsis como los descritos por Pertuz et $\mathrm{al}^{19}$ en Santa Marta (Colombia), casos que estaban ubicados en unidades de cuidados intensivos; en los que se concluyó que "la sepsis provenía de la comunidad y/o asociada a la atención en salud".

Se valoró la adherencia a la norma institucional de "no uso de joyas" en los funcionarios evaluados en la tercera fase, con un $86,7 \%$, lo que constituye un mejor logro que el descrito por Scott ${ }^{20}$, estudio en el cual el 36,7\% del personal llevaba anillo; el 46,9\%, reloj y el 6,1\%, pulsera $^{8,12,15}$. De la misma manera, la adherencia al "no uso de esmaltes" con logros del $91,8 \%$ en la tercera fase, es un resultado satisfactorio. En una investigación publicada sobre la práctica del lavado de manos en un hospital en Honduras, la mayoría de los encuestados $(82,4 \%)$ refirieron que el uso de esmaltes y uñas artificiales son medios de transferencia de bacterias ${ }^{21-23}$. 


\section{CONCLUSIÓN}

Se analizó la adherencia al lavado de las manos según los cinco Momentos de la Higiene de Manos de la OMS; los resultados se compararon con estudios similares, ejercicio que arrojó, para los componentes del $1^{\circ}$ al $4^{\circ}$, mejores porcentajes de adherencia en el presente estudio que en otros como el del Hospital General Universitario de Alicante. Sin embargo, respecto al $5^{\circ}$ momento de la OMS, no puede concluirse lo mismo por no existir estudios para hacer el paralelo. La omisión de este paso en investigaciones similares hace de este análisis uno más completo al tener en cuenta la totalidad de los Momentos de la OMS.

En suma, se debe seguir trabajando para mejorar la concientización en la práctica del lavado de las manos, para asegurar su continuidad y fortalecimiento. Se debe insistir en el buen uso de los guantes y de otras normas que buscan la prevención de infecciones, como inducción y re-inducción al personal asistencial para mantener las uñas cortas y sin esmalte; el retiro de relojes, pulseras, anillos, argollas, manillas, dejando libres las muñecas y zonas vecinas para su adecuada higiene, sin importar el material del que estén elaborados; no aplazar el recambio del esmalte por más de cuatro días porque el esmalte caduco alberga más bacterias; usar uniformes de manga corta y cabello recogido por encima del cuello.

Todas las anteriores son medidas complementarias que hacen parte de las recomendaciones para los funcionarios asistenciales de Assbasalud ESE y que pueden extenderse a todas las IPS. Este es un paso más del programa de seguridad del paciente de la entidad objeto de estudio, que ha propiciado progresos en la "cultura de seguridad del paciente" gracias a la implementación de estrategias en favor de la prevención integral de las infecciones intrahospitalarias, que incluyen la aplicación de protocolos y guías, complementadas con Políticas de Calidad y Políticas de Seguridad del paciente, de cuyo seguimiento se encargan los comités de Seguridad del Paciente y Ejecutivo de Calidad. A futuro, todas estas acciones pueden ser tema de nuevas investigaciones.

\section{DECLARACIÓN SOBRE CONFLICTOS DE INTERESES}

Los autores de este manuscrito declaran no poseer conflicto de interés asociado con contratación, consultoría, inversión, financiación, relación familiar o con los resultados de la investigación.

\section{REFERENCIAS BIBLIOGRÁFICAS}

1. Miranda M, Navarrete L. Semmelweis y su aporte científico a la medicina: Un lavado de manos salva vidas. Rev. chil. Infectol. 2008; 25 (1): 54-7.

2. Organización Mundial de la Salud. Manual técnico de referencia para la higiene de las manos. Informe de un grupo científico de la OMS. Ginebra: OMS; 2009.

3. Organización Mundial de la Salud. Guía de la OMS sobre higiene de manos en la atención de la salud. Primer Desafío Global de la Seguridad del Paciente Atención Limpia es Atención Segura. Informe grupo científico de la OMS. Ginebra: OMS; 2009.

4. Organización Mundial de la Salud. Alianza mundial para la seguridad del paciente: mayor conocimiento para atención más segura. Informe Grupo Científico de la OMS. Ginebra: OMS; 2008.

5. Hernández LR, Gámez A, Gómez A. Consideraciones sobre la sepsis intrahospitalaria en un servicio de geriatría. Rev Cubana Enfermer. 2005; 21 (2).

6. Maciel SF, Padilha T, Kuerten P, Lessmann JC. Higienização das mãos e a segurança do paciente pediátrico. Cienc. enferm 2013; 19 (2): 99-109.

7. Aparecida PM, Anselmi ML, Dalmas JC. Evaluación de la calidad de los procedimientos curativos en pacientes internados en un hospital universitario. Rev Latino-Am. Enfermagem. 2008; 16(1): 57-63.

8. Ardila AM, Muñoz AI. Bioseguridad con énfasis en contaminantes biológicos en trabajadores de la salud. Ciênc saúde coletiva. 2009; 14 (6): 2135-41.

9. Toribio R. Higiene de manos en los centros sanitarios. Documentos para directivos y responsables de la higiene de manos. Madrid: Servicio Extremeño de Salud; 2013

10. Friedman C, Newsom W. Conceptos básicos de control de infecciones de International Federation of Infection Control. Segunda edición. Portadown: IFIC; 2011.

11. Troconis JE. El lavado y cuidado de las manos. Acta odontol Venez. 2003; 41 (2): 166-71.

12. Colombia. Ministerio de Salud. Resolución No ${ }^{\circ} .8430$ de 1993 que establece las normas científicas, técnicas y administrativas para la investigación en salud. Diario Oficial. (Octubre 4 de 1993).

13. 59a Asamblea General. Declaración de Helsinki de la Asociación Médica Mundial. Principios éticos para las investigaciones médicas en seres humanos. Seúl: Asociación Médica Mundial; 2008.

14. Zamudio I, Meza A, Martínez Y. Estudio multimodal de higiene de manos en un hospital pediátrico de tercer nivel. Bol Med Hosp Infant Mex. 2012; 69 (5): 384-390.

15. Sánchez J, Fuster M, García C, Gracia RM. Evaluación de las recomendaciones sobre la higiene de manos. Anales Sis San Navarra. 2007; 30(3): 343-52. 
16. Instituto Nacional de Salud Pública. Cumplimiento de la higiene de manos según la OMS y factores asociados al personal de salud de pediatría de un hospital general de la Secretaría de Salud. México DF: Instituto Nacional de Salud Pública; 2010.

17. Pessoa CL, Hugonnet S, Pfister R, Touveneau S, Dharan $\mathrm{S}$, Posfay $\mathrm{K}$, et àl. Reduction of Health Care-Associated Infection Risk in Neonates by Successful Hand Hygiene Promotion. Pediatrics. 2007; 120 (2): 382-90.

18. Acosta S, De Andrade V. Manual de esterilización para centros de salud. Organización Panamericana de SaludUSAID. Washington DC: OPS; 2008.

19. Pertuz Y, Pérez C, Pabón Y. Aspectos epidemiológicos de la sepsis, en unidades de cuidados intensivos Santa Marta, Colombia. Revista Duazary. 2016; 13 (2):126-32.
20. Scott S. Hand Washing, Hygiene, CGMP, and Science. Journal of GXP Compliance. 2010; 14 (1): 62-69.

21. Universidad Nacional Autónoma de Honduras. Práctica de lavado de manos en Ginecología y Pediatría, Hospital Aníbal Murillo Escobar. Unidad de Investigación Científica. Facultad de Ciencias Médicas. Tegucigalpa: UNAH; 2011.

22. Ministerio de Salud Pública y Asistencia Social. Lineamientos técnicos en la prevención y control de infecciones. Manual de Enfermería. Dirección de Enfermería. Minsalud Pública. San Salvador; 2006.

23. Ministerio de Salud. Dirección de Salud del Cusco. Prevención de infecciones intrahospitalarias. Guía de lavado de manos. Cusco: Dirección de epidemiologia; 2011.

Para citar este artículo: Villegas-Arenas O, Gómez J, López J, Román R, Villa J, Botero $\mathrm{J}$, et al. Medición de la adherencia al lavado de manos, según los cinco momentos de la OMS. Duazary. 2017 julio; 14 (2): 169 - 178. Doi: http://dx.doi.org/10.21676/2389783X.1967 